\title{
Un modelo MILP multiperíodo para el diseño de una cadena de suministro de bioetanol considerando sustentabilidad
}

\author{
Alejandra Gagliardo ${ }^{1}$, Gabriela Corsano ${ }^{1,2}$ \\ ${ }^{1}$ Departamento de Matemática, Facultad de Ingenieía Química (FIQ), Universidad Nacional \\ del Litoral (UNL), Santiago del Estero 2928, Santa Fe, Argentina. \\ ${ }^{2}$ Instituto de Desarrollo y Diseño (INGAR), Avellaneda 3657 S3002GJC Santa Fe, \\ Argentina. alegagliardo@hotmail.com,gcorsano@santafe-conicet.gov.ar
}

\begin{abstract}
Resumen. En este trabajo se propone un modelo matemático mixto entero lineal para el diseño óptimo de una cadena de suministro para producir azúcar y etanol. La formulación considera varios períodos de producción-inventariodistribución y restricciones ambientales. El impacto ambiental es incorporado en este modelo mediante los principios del Análisis de Ciclo de Vida, derivando en un problema de programación matemática multi-objetivo. Mediante la resolución sucesiva de modelos, donde se optimiza el objetivo económico y se manejan las restricciones ambientales mediante el método " $\varepsilon$-constraint", se obtienen diferentes soluciones del tipo Pareto. Este enfoque permite evaluar diferentes escenarios de integración y sirve como guía en la toma de decisiones al momento de diseñar una cadena de suministro sustentable.
\end{abstract}

Palabras Claves: Modelación matemática, optimización multi-objetivo, cadenas de suministros, sustentabilidad.

\section{Introducción}

Las reservas de combustibles fósiles se están agotando, el calentamiento global se está convirtiendo en una realidad, el reciclado de residuos es cada vez mayor, más costoso y problemático, y el inexorable crecimiento de la población requiere más y más energía y alimentos. Frente a este panorama, la producción de los biocombustibles surge como una solución a muchos de los problemas socio-económico-ambientales actuales. En particular, la caña de azúcar es una especie a partir de la cual el bioetanol se produce eficientemente en el mundo [1]. Por otro lado, el análisis de sustentabilidad es una herramienta cada vez más presente en el análisis social y político de las alternativas técnicas de desarrollo industrial o de mejoramiento de los procesos ya instalados, y se está transformando en un nuevo paradigma que influenciará a las ingenierías que requiere para su evaluación.

Grossmann y Guillén-Gosálbez [2] presentaron un "review" de las contribuciones realizadas por diferentes autores sobre programación matemática para la síntesis y el 
planeamiento de procesos sustentables. Ellos centraron su trabajo en dos áreas específicas de ingeniería de procesos: síntesis de procesos y cadenas de suministros (CS). Estos mismos autores presentaron un modelo mixto entero no lineal (MINLP) para el diseño y planeamiento de una CS sustentable bajo incertidumbre [3].

Zamboni y col. [4] formularon un modelo matemático para la optimización de una CS para la producción de bioetanol a partir de granos, donde la función objetivo considera la minimización de los costos de capital, producción y transporte. En un trabajo posterior [5], incorporaron consideraciones ambientales en la función económica tradicional, dando una herramienta de optimización multi-objetivo.

Recientemente Mele y col. [6] presentaron un modelo mixto entero lineal (MILP) para el diseño óptimo de una CS para la producción de azúcar y etanol, considerando el impacto ambiental provocado por las etapas de cultivo de caña, producción de azúcar y etanol, y transporte de materias primas y productos finales. Las ventajas del enfoque presentado son mostradas a través de un ejemplo de dicha industria en Argentina.

Corsano y col. [7] presentaron un modelo MINLP para el diseño óptimo de una CS para la producción de etanol y levaduras, considerando el diseño detallado de las plantas de producción de etanol y derivados. Los autores analizaron la disposición de residuos mediante penalidades en la función objetivo. El modelo obtiene diferentes escenarios donde el diseño de la CS y de las plantas involucradas varían de acuerdo a la política que se adopte para el desecho de residuos y/o la producción de levaduras a partir de éstos.

Wang y col. [8] presentan un modelo de optimización multi-objetivo para el diseño de una CS considerando el impacto ambiental como una nueva categoría de variables de decisión, denominada nivel de protección ambiental. Los autores además presentan un análisis de sensitividad para el caso de estudio, donde concluyen que mejorando la capacidad de la red e incrementando el suministro en las instalaciones, las emisiones de $\mathrm{CO}_{2}$ y el costo total pueden decrecer en la red global.

Pinto-Varela y col. [9] presentan un enfoque bi-criterio para el diseño y planeamiento óptimo de una CS, donde los aspectos ambientales son considerados a través de la metodología Eco-indicator. Proponen un modelo lineal basado en programación difusa (fuzzy programming) para balancear el beneficio con el impacto ambiental, mientras que la CS es modelada mediante un MILP.

En este trabajo se presenta un modelo MILP para el diseño óptimo sustentable de una CS para la producción de etanol a partir de las melazas obtenidas del proceso de producción de azúcar de caña. La CS considera cuatro niveles: plantas de azúcar que representan sitios de materia prima, plantas de producción de etanol, depósitos de azúcar y etanol, y clientes. El modelo determina qué plantas de etanol y depósitos de etanol y azúcar instalar, los flujos entre los distintos nodos de la cadena en cada período, el inventario en cada depósito en cada período, y el número de camiones que se utilizará para la distribución de materia prima, productos y residuos en cada período, con el fin de satisfacer las demandas mínimas y máximas de los clientes en cada período a un máximo beneficio y considerando el impacto ambiental provocado por la producción y distribución de los productos. Las vinazas producidas en las plantas de etanol son desechadas en los ríos cercanos y se calcula el impacto ambiental correspondiente. La performance ambiental de la CS se toma en base a los índices de impacto de acuerdo a los principios del Análisis de Ciclo de Vida (LCA) 
teniendo en cuenta las etapas de producción de azúcar y etanol, y de transporte de melazas, productos y vinazas. Se adoptan los índices del potencial de calentamiento global propuestos por Mele y col. [6] usados para esta industria.

El modelo se presenta como una formulación multi-objetivo y se resuelve optimizando en forma iterativa el modelo económico donde el daño ambiental es considerado mediante restricciones del tipo $\varepsilon$-constraint, para distintos valores mínimos y máximos del parámetro $\varepsilon$. De esta manera se obtiene un abanico de soluciones que representan diferentes escenarios económicos-ambientales para la cadena propuesta.

El modelo es aplicado a la producción de azúcar actual del norte provincial santafesino, donde se conocen posibilidades de expansión de la producción de caña de azúcar [10]. En base a estos datos y utilizando los parámetros de costos y ambientales adoptados por Mele y col. [6], se optimiza el diseño de la CS de biocombustibles regional.

\section{Descripción del Problema}

La Figura 1 muestra el esquema de la CS propuesta para la producción y distribución de azúcar y etanol. Las plantas de azúcar producen azúcar para satisfacer las demandas de los clientes, y melazas destinadas a la producción de etanol. Cada planta de etanol produce además, un residuo denominado vinazas, las cuales serán descartadas a los ríos más cercanos. Las plantas de azúcar se encuentran ya instaladas en lugares conocidos, mientras que la instalación de las plantas de etanol es una variable de optimización.

Los productos (azúcar y etanol) se depositan en diferentes almacenes y se permite almacenar producto de un mes a otro. La instalación de los depósitos es una decisión del problema y el número de depósitos a instalar es acotado.

Los clientes se encuentran en lugares conocidos y sus demandas mínimas y máximas deben ser satisfechas en cada período.

Los flujos entre los diferentes nodos de la CS en cada período es una variable de decisión y se disponen de vehículos de diferentes capacidades para transportar las melazas, azúcar, etanol y vinazas. El número y tipo de vehículo que se debe utilizar para transportar el material entre los diferentes nodos de la cadena es una variable de optimización del modelo.

El impacto ambiental se mide a través del potencial del calentamiento global (GWP100) en kilogramo de dióxido de carbono por kilogramo de emisión a escala mundial y se aplica al sistema de producción de azúcar y etanol, y al sistema de transporte de los diferentes materiales. Los coeficientes para dichos sistemas son los presentados por Mele y col. [6] para las tecnologías T1 (producción de azúcar y melaza) y T3 (producción de etanol a partir de melazas).

En resumen, el problema de diseño óptimo sustentable de la CS de etanol propuesta en este trabajo se define como:

Conociendo

- la caña disponible en cada período en las cercanías de las plantas de azúcar disponibles 
- los factores de producción de azúcar y melazas

- los factores de conversión de melaza en etanol y vinazas

- el número máximo y capacidad máxima de plantas de etanol y depósitos de etanol y azúcar a instalarse

- las demandas mínimas y máximas de cada producto para cada cliente

- el número máximo de vehículos disponibles en cada instalación y sus capacidades

- los precios de los productos

- los costos de instalación, producción, mantenimiento y transporte,

- los parámetros correspondientes a los factores de impacto ambiental Determinar

- la configuración de la CS: la instalación de plantas de etanol y depósitos de azúcar y etanol

- la producción de etanol en cada planta en cada período

- el inventario de etanol en cada depósito en cada período

- el inventario de azúcar en cada depósito en cada período

- las vinazas producidas en las plantas instaladas en cada período

- los flujos entre los distintos nodos de la CS en cada período, y

- el número y tipo de vehículo utilizados entre los distintos nodos de la cadena en cada período

con el fin de maximizar el valor presente neto (ganancias menos costos), minimizando el impacto ambiental provocado por la producción de azúcar y etanol y el transporte de todos los flujos involucrados en la red global.

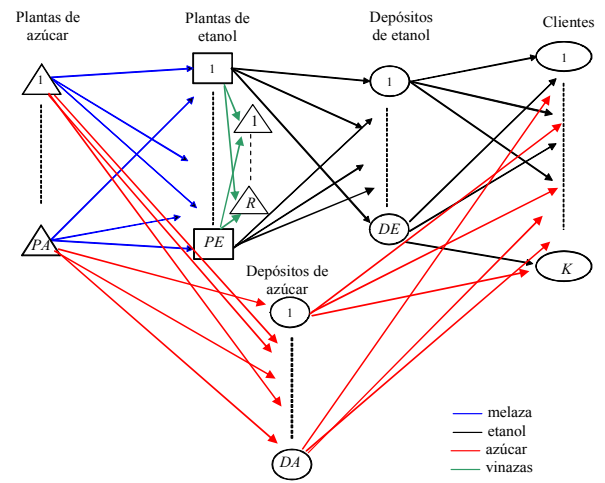

Fig. 1. Esquema de la cadena de suministro

\section{Modelo Matemático}

En esta sección se describe el modelo matemático formulado para el problema presentado anteriormente. Sea $P A$ el número de plantas de azúcar instaladas ( $p a=$ $1, \ldots, P A), P E$ el número máximo de plantas de etanol a instalarse $(p e=1, \ldots, P E), D A$ y $D E$ el número máximo de depósitos de azúcar y etanol a instalarse respectivamente 
$(d a=1, \ldots, D A ; d e=1, \ldots, D E)$, y $K$ el número de clientes $(k=1, \ldots, K)$. Las vinazas producidas en cada planta pe se trasladan a los ríos $r=1, \ldots, R$. Además, se definen las variables binarias de asignación: $x_{p e}$ para determinar la instalación de la planta $p e$, $y_{d e}$ y $z_{d a}$ para la asignación del depósito de y $d a$ respectivamente. Para transportar cada producto y subproducto $i$ ( $i=$ melaza, azúcar, etanol y vinazas) de un nodo a otro de la CS en cada período $t(t=0, \ldots, T)$, se utilizan camiones del tipo $l i$ con capacidades distintas, $l i=1, \ldots, L i$. En este caso, se considera que los camiones de tipo $l 1$ son utilizados para el transporte de melazas, los de tipo $l 2$ para el transporte de azúcar, los de tipo $l 3$ para el transporte de etanol y los de tipo $l 4$ para el transporte de vinazas. Luego, el modelo considera las restricciones que se plantean a continuación:

\subsection{Balances de masa}

En las cercanías de cada planta de azúcar instalada existe una cantidad conocida de caña de azúcar para moler en cada período $t, C M_{p a, t}$, de tal manera que la cantidad de azúcar y melaza disponible en cada período es:

$$
\begin{array}{cl}
A z_{p a, t}=v_{a z} C M_{p a, t} & \forall p a, t \geq 1 \\
M e l_{p a, t}=\rho_{\text {miel }} C M_{p a, t} & \forall p a, t \geq 1
\end{array}
$$

donde $v_{a z}$ y $\rho_{\text {miel }}$ son factores de conversión de caña-azúcar y caña-melaza respectivamente.

Luego, como la cantidad de melaza obtenida en cada período es utilizada para la obtención de etanol, las ecuaciones para el consumo de la misma en las plantas de etanol son:

$$
\begin{gathered}
\alpha_{m i e l} \sum_{p a, l 1} Q M_{p a, p e, l 1, t}=Q E T_{p e, t} \quad \forall p e, t \geq 1 \\
\sum_{p e, l 1} Q M_{p a, p e, l 1, t} \leq M e l_{p a, t} \quad \forall p a, t \geq 1 \\
Q M_{p a, p e, l 1, t} \leq M e l_{p a, t} x_{p e} \quad \forall p a, p e, l 1, t \geq 1
\end{gathered}
$$

donde $Q M_{p a, p e, l 1, t}$ representa las toneladas de melaza transportada desde $p a$ a pe en vehículos del tipo $l 1$ en el período $t, Q E T_{p e, t}$ la producción total (en ton) de etanol en la planta pe en el período $t, \alpha_{\text {miel }}$ representa el factor de conversión de melaza-etanol. La ecuación (5) representa una cota superior para el transporte de melaza a la planta pe si dicha planta se instala y obliga a que sea cero este transporte si la planta no se instala.

Los balances entre las plantas y los depósitos vienen dados por las ecuaciones:

$$
\sum_{d a, l 2} Q A_{p a, d a, l 2, t} \leq A z_{p a, t} \quad \forall p a, t \geq 1
$$




$$
\sum_{d e, l 3} Q E_{p e, d e, l 3, t}=Q E T_{p e, t} \quad \forall d e, t \geq 1
$$

donde $Q A_{p a, d a, l 2, t}$ representa el azúcar transportada de $p a$ a $d a$ en vehículos del tipo $l 2$ en el período $t$, y $Q E_{p e, d e, 1, t, t}$ representa el etanol transportado de $p e$ a de en vehículos del tipo $l 3$ en el período $t$.

Las vinazas producidas en cada planta de etanol $(Q V T)$ son transportadas y desechadas en los ríos $(r=1, \ldots, R)$ de la región $\left(Q V_{p e, r, 14, t}\right)$, utilizando vehículos del tipo $l 4$ de diferentes capacidades. Este desecho tendrá un impacto ambiental asociado en las restricciones del LCA. Las ecuaciones de producción y traslado de vinazas son las siguientes:

$$
\begin{aligned}
& Q V T_{p e, t}=\beta_{v i n} Q E T_{p e, t} \quad \forall p e, t \geq 1 \\
& \sum_{r, l 4} Q V_{p e, r, l 4, t}=Q V T_{p e, t} \quad \forall p e, t \geq 1
\end{aligned}
$$

donde $\beta_{v i n}$ es el factor de conversión de etanol-vinazas.

\subsection{Balances de inventario}

Los productos son trasladados de las plantas a los depósitos y luego pueden ser almacenados o enviados a los clientes en cada período. Si dichos productos son almacenados, las ecuaciones de balancesde inventario son las siguientes:

$$
\begin{aligned}
& I N V A_{d a, t-1}+\sum_{p a, l 2} Q A_{p a, d a, l, t, t}=I N V A_{d a, t}+\sum_{k, l 2} Q A K_{d a, k, l 2, t} \quad \forall d a, t \\
& I N V E_{d e, t-1}+\sum_{p e, l 3} Q E_{p e, d e, l 3, t}=I N V E_{d e, t}+\sum_{k, l 3} Q E K_{d e, k, l 3, t} \quad \forall d e, t \\
& I N V A_{d a, t} \leq C D A_{d a}^{U P} z_{d a} \quad \forall d a, t \\
& I N V E_{d e, t} \leq C D E_{d e}^{U P} y_{d e} \quad \forall d e, t \\
& Q A K_{d a, k, l 2, t} \leq C D A_{d a}^{U P} z_{d a} \quad \forall d a, t \\
& Q E K_{d e, k, l 3, t} \leq C D E_{d e}^{U P} y_{d e} \quad \forall d e, t \\
& I N V A_{d a, t}=0 \quad \forall d a, t=0, t=T \\
& I N V E_{d e, t}=0 \quad \forall d e, t=0, t=T
\end{aligned}
$$


donde $I N V A_{d a, t}$ e $I N V E_{d e, t}$ representan las toneladas de azúcar y etanol respectivamente, almacenadas en cada depósito al final del período $t, Q A K_{d a, k, l 2, t}$ y $Q E K_{d e, k, l 3, t}$ son las toneladas transportadas de azúcar y etanol respectivamente al cliente $k$ en el período $t$ en camiones de tipo $l i$, y $C D A_{d a}^{U P}$ y $C D E_{d e}^{U P}$ son las capacidades máximas de los depósitos de azúcar y etanol respectivamente. Las ecuaciones (14) y (15) establecen que no se cuenta con un stock inicial y que se requiere que el stock sea cero al terminar el horizonte de producción.

\subsection{Restricciones de demanda}

Cada cliente tiene demandas mínimas y máximas de cada producto en cada período: $D e m A_{k}^{L O}, D e m A_{k}^{U P}, D e m E_{k}^{L O}$ y $\operatorname{Dem} E_{k}^{U P}$ para azúcar y etanol respectivamente. La satisfacción de estas demandas se expresan mediante las siguientes restricciones:

$$
\begin{aligned}
& \operatorname{Dem}_{k}^{L O} \leq \sum_{d a, l 2} Q A K_{d a, k, l 2, t} \leq \operatorname{Dem}_{k}^{U P} \quad \forall k, t \geq 1 \\
& \operatorname{DemE}_{k}^{L O} \leq \sum_{d e, l 3} Q E K_{d e, k, l 3, t} \leq \operatorname{DemE}_{k}^{U P} \quad \forall k, t \geq 1
\end{aligned}
$$

\subsection{Cálculo del número de camiones de cada tipo}

En este enfoque se considera al número de vehículos utilizados como una variable continua. En un futuro trabajo, se estudiará el tratado de estas variables como discreta, lo cual implica incorporar un gran número de variables binarias que incrementa la complejidad computacional del modelo.

Sea $N C_{p a, p e, 11, t}$ la variable continua que denota el número de camiones de tipo $l 1$ usados para transportar melaza desde $p a$ hacia pe en el período $t$, luego, el cálculo para determinar el número de camiones a utilizar para dicho transporte se representa en la restricción (18):

$$
\begin{gathered}
N C_{p a, p e, l 1, t} \geq \frac{Q M_{p a, p e, l 1, t}}{C A P_{l 1}} \quad \forall p a, p e, l 1, t \geq 1 \\
\sum_{p e=1}^{P E} N C_{p a, p e, l 1, t} \leq N C 1_{l 1, p a}^{U P} x_{p e} \quad \forall p a, l 1, t \geq 1
\end{gathered}
$$

La restricción (19) establece que no pueden ser usados más de $N C 1_{l 1, p a}^{U P}$ vehículos de capacidad $l l$ si la planta pe se instala y obliga a que tome valor cero la variable $N C_{\text {pa,pe,l1,t }}$ si la planta no se instala.

Análogamente se calcula el número de camiones desde $p a$ a $d a$ y desde $d a$ a $k$ de tipo 12 ; desde pe a de y de de a $k$ de tipo 13 ; y desde pe a $r$ de tipo $l 4$. 


\subsection{Restricciones correspondientes al LCA}

Como se mencionó previamente, en este trabajo se considera la métrica GWP100 para determinar el daño ambiental causado por la producción y transporte de azúcar y etanol. Para el cálculo de este daño se considera el inventario de emisiones expresado a través de las producciones de azúcar y etanol para la etapa de producción, y los flujos de la CS para el transporte. En este modelo se considera entonces:

$$
D A \tilde{N} O=\sum_{b, t} v_{b} I A_{b t}
$$

donde $v_{b}$ es el factor de daño de la especie $b$ (obtenido de la métrica GWP100) e $I A_{b t}$ es el daño ambiental para la especie $b$ en el período $t$. Por ejemplo para la producción y transporte de azúcar $I A_{a z, t}$ es:

$$
I A_{a z, t}=\sum_{p a} f p_{a z} A z_{p a, t}+\sum_{p a, d a, l 2} f t_{a z} N C_{p a, d a, l 2, t} d i s_{p a, d a}+\sum_{d a, k, l 2} f t_{a z} N C_{d a, k, l 2, t} d i s_{d a, k}
$$

donde $d i s_{a, b}$ representa la distancia entre $a$ y $b$, y $f p$ y $f t$ representan los factores de emisión del efecto invernadero provocado por la producción y transporte de azúcar respectivamente. Análogamente se calcula el impacto provocado por la producción y transporte de etanol, y por el transporte de melazas y vinazas.

\subsection{Ecuaciones económicas}

La función económica considerada es la maximización del valor presente neto dado por:

$$
V P N=\sum_{t=1}^{T} \frac{\text { Gan }_{t}}{(1+r)^{t-1}}
$$

donde $r$ es la taza de interés y $\operatorname{Gan}_{t}$ representa el ingreso por venta de azúcar $\left(I V_{a z, t}\right)$ y etanol $\left(I V_{e t, t}\right)$, menos los costos de instalación de plantas y depósitos $\left(C I_{t}\right.$ igual al costo de inversión total dividido el número de períodos), producción de azúcar y etanol $\left(C P_{t}\right)$, mantenimiento del inventario $\left(C M_{t}\right)$ y transporte de melazas, azúcar, etanol y vinazas $\left(C T_{t}\right)$ :

$$
G a n_{t}=I V_{a z, t}+I V_{e t, t}-\left(C I_{t}+C P_{t}+C M_{t}+C T_{t}\right) \quad \forall t
$$

Por falta de espacio no se muestran las ecuaciones correspondientes a cada costo, pero son calculados mediante coeficientes de costos multiplicados por las cantidades producidas o almacenadas para el caso de la producción y mantenimiento de inventario respectivamente, costos fijos para los costos de instalación de plantas de etanol y depósitos, y en el caso de transporte multiplicando el costo del combustible 
(\$/1) por el número de vehículos utilizados por la distancia $(\mathrm{km})$ dividido el rendimiento $(\mathrm{km} / \mathrm{l})$ del vehículo utilizado.

\subsection{Función Objetivo}

Como se mencionó anteriormente, el objetivo del enfoque propuesto es hallar el diseño óptimo de la CS con mayor ganancia y menor impacto ambiental. De esta forma, el problema resulta en un modelo multi-objetivo que puede plantearse en general como:

$$
\begin{array}{r}
\min _{x, y}\{-V P N(x, y) ; D A \tilde{N} O(x, y)\} \\
\text { sujeta a: restricciones }(1)-(23) \\
x \in \mathfrak{R}, y \in\{0,1\}
\end{array}
$$

donde $x$ e $y$ representan las variables continuas y discretas del modelo.

Se propone resolver el modelo generando puntos del tipo Pareto considerando las ecuaciones ambientales dentro del conjunto de restricciones y acotando la variable $D A \tilde{N} O$ entre dos valores $\left(\varepsilon_{\min }, \varepsilon_{\max }\right)$ que se obtienen mediante la resolución de dos sub-problemas [6]. De esta forma, el problema maestro que se resuelve es:

$$
\max _{x, y} \operatorname{VPN}(x, y)
$$

sujeta a : restricciones $(1)-(23)$

$$
\begin{gathered}
\mathcal{E}_{\min } \leq D A \tilde{N} O \leq \varepsilon_{\max } \\
x \in \Re, y \in\{0,1\}
\end{gathered}
$$

donde $\varepsilon_{\min }$ toma el valor óptimo del $D A \tilde{N} O$ del problema:

$$
\min _{x, y} D A \tilde{N} O(x, y)
$$

sujeta a : restricciones $(1)-(23)$

$$
x \in \Re, y \in\{0,1\}
$$

mientras que $\varepsilon_{\max }$ toma el valor óptimo de la variable $D A \tilde{N} O$ del problema:

$$
\max _{x, y} \operatorname{VPN}(x, y)
$$

sujeta a : restricciones $(1)-(23)$

$$
x \in \Re, y \in\{0,1\}
$$

Para la obtención de los puntos de tipo Pareto, se resuelve en forma iterativa el problema maestro, acotando el valor del parámetro $\varepsilon$ con valores más grandes que la cota mínima obtenida en el sub-modelo (26) y más chicos que la cota máxima obtenida en el sub-modelo (27). Las soluciones óptimas obtenidas de la resolución de cada modelo maestro representan las distintas soluciones Pareto. 


\section{Caso de estudio}

Se desea obtener el diseño óptimo sustentable de una CS para la producción de etanol y azúcar en la zona centro-norte de la provincia de Santa Fe, tomando como horizonte de producción un año, el cual se divide en 4 períodos (trimestres). Se consideran 2 plantas de azúcar, ubicadas en las localidades las Toscas y Villa Ocampo en el departamento General Obligado, y 5 clientes con demandas máximas de etanol y azúcar como se muestra en la Tabla 1 para todos los períodos. Las demandas mínimas de ambos productos para todos los clientes igual a cero en todos los períodos.

Se evalúa, además, la instalación de hasta 5 plantas de etanol y 5 depósitos de etanol y azúcar respectivamente de distintas capacidades, distribuidos geográficamente en 4 departamentos (regiones): General Obligado, Vera, San Justo y La Capital, como se observa en la Figura 2.

Debido a que las dos plantas de azúcar instaladas se encuentran en el departamento General Obligado, se localizan 2 posibles instalaciones de plantas y depósitos de etanol como así también 2 posibles instalaciones de depósitos de azúcar.

La Tabla 2 muestra la cantidad de caña de azúcar disponible en cada período en las cercanías de cada planta de azúcar. Mediante los factores de conversión caña-azúcar y caña-melaza, que fueron extraídos del trabajo de Mele y col. [6], pueden calcularse las cantidades máximas a producir de azúcar y melazas en cada planta y se muestran en la Tabla 2.

El número máximo de camiones disponibles en cada instalación en cada período es 60 para el transporte de melazas de capacidades 15,20 y 30 ton, 180 para vinazas de capacidades 20, 25 y 30 ton, 120 para azúcar de capacidades 20, 25 y 30 ton y 30 para etanol de capacidades 5, 10 y 20 ton. El rendimiento que tienen los camiones es 10, 8 y $5 \mathrm{~km}$ por litro de combustible para los camiones pequeños, medianos y grandes respectivamente para cada tipo $l i$, mientras que el precio por litro de combustible es de \$5 para todos los camiones utilizados en el transporte de los diferentes productos.

La Tabla 3 muestra las distancias entre las distintas regiones, considerando como regiones 1 y 2 a las instalaciones que se encuentran cercanas a los ingenios de Las Toscas e Inaza respectivamente, y regiones 3, 4 y 5 a las zonas de las posibles instalaciones restantes, enumeradas de norte a sur. Las vinazas producidas en las plantas de etanol serán desechadas a los ríos Paraná y Salado. Las distancias entre las diferentes regiones y estos ríos se muestran en la Tabla 4.

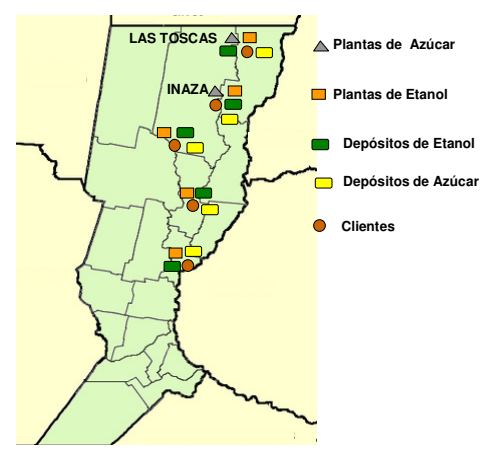

Fig. 2. Distribución geográfica de los nodos de la CS para el caso de estudio 
Tabla 1. Demandas Máximas de Etanol y Azúcar (ton) por período

\begin{tabular}{lll}
\hline Clientes & Etanol & Azúcar \\
\hline 1 & 175 & 1750 \\
2 & 105 & 1050 \\
3 & 105 & 1050 \\
4 & 140 & 1400 \\
5 & 350 & 3500 \\
\hline
\end{tabular}

Tabla 2. Cantidades máximas disponibles en cada planta de azúcar (ton)

\begin{tabular}{llll}
\hline Períodos & $\begin{array}{l}\text { Caña de } \\
\text { Azúcar }\end{array}$ & Azúcar & Melaza \\
\hline 1 & 31490 & 3778.9 & 1556.86 \\
2 & 62980 & 7557.6 & 3113.73 \\
3 & 15745 & 1889.4 & 778.43 \\
4 & 15745 & 1889.4 & 778.43 \\
\hline
\end{tabular}

Tabla 3. Distancia en km entre las distintas regiones

\begin{tabular}{llllll}
\hline & Región 1 & Región 2 & Región 3 & Región 4 & Región 5 \\
\hline Región 1 & 0 & 25 & 217 & 325 & 425 \\
Región 2 & 25 & 0 & 192 & 300 & 400 \\
Región 3 & 217 & 192 & 0 & 108 & 208 \\
Región 4 & 325 & 300 & 108 & 0 & 100 \\
Región 5 & 425 & 400 & 208 & 100 & 0 \\
\hline
\end{tabular}

Tabla 4. Distancia en km entre las distintas regiones y los ríos.

\begin{tabular}{lll}
\hline Plantas de etanol & Río Paraná & Río Salado \\
\hline 1 & 20 & 220 \\
2 & 30 & 180 \\
3 & 80 & 20 \\
4 & 80 & 20 \\
5 & 20 & 3 \\
\hline
\end{tabular}

Los precios de venta de azúcar son $644,537,708$ y 708 \$/ton para los períodos 1 a 4 respectivamente, mientras los precios de venta de etanol son 1032, 860, 1135 y 1135 \$/ton para los períodos 1 al 4 respectivamente. Los costos de instalación de los depósitos de etanol y azúcar son de $\$ 5000$ y $\$ 10000$ respectivamente, mientras que las capacidades máximas de los depósitos son de 500 ton para etanol y 5000 ton para azúcar. Los costos de producción e inventario como así también los parámetros para el impacto ambiental fueron extraídos del trabajo de Mele y col. [6]. El modelo fue formulado y resuelto en GAMS [11], con el resolvedor Cplex, en una PC Intel (R) 
Core2, 1.86 Ghz. El modelo de optimización contiene 3389 ecuaciones, 2782 variables continuas y 15 variables discretas y fue resuelto en 1.3 segundos.

Se resuelve en primera instancia los dos subproblemas (26) y (27) para obtener los valores de $\varepsilon_{\min }$ y $\varepsilon_{\max }$ respectivamente.

La solución que se obtiene del problema (26) corresponde a un valor de $\varepsilon_{\min }$ igual a 1200500. Esta solución consiste en no instalar ninguna planta de etanol, debido al fuerte impacto que provoca esta producción. Como las plantas de azúcar están instaladas y procesan toda la caña disponible, la solución del problema (26) selecciona tres depósitos de azúcar (región 1, 2 y 5) para la distribución de este producto a los clientes. Cabe mencionar que el VPN de esta solución es igual a cero, ya que para minimizar el impacto distribuye la menor cantidad posible de azúcar producida. La mejor solución ambiental corresponde a no distribuir nada de azúcar, pero debido a que la variable $V P N$ es no negativa, distribuye la menor cantidad para satisfacer esta condición. En cambio, la solución óptima del problema (27) consiste en la instalación de dos plantas de etanol (región 1 y 2), las cuales procesan toda la melaza disponible en cada período. Esto ocurre porque no se tiene en cuenta el daño ambiental y como la producción de etanol es rentable, produce la cantidad máxima de este producto. El valor del daño ambiental de esta solución representa la cota superior para el daño del problema (25) y es $\varepsilon_{\max }=32768000$.

La solución óptima del problema (25) consiste en el diseño de la CS que se muestra en la Figura 3.

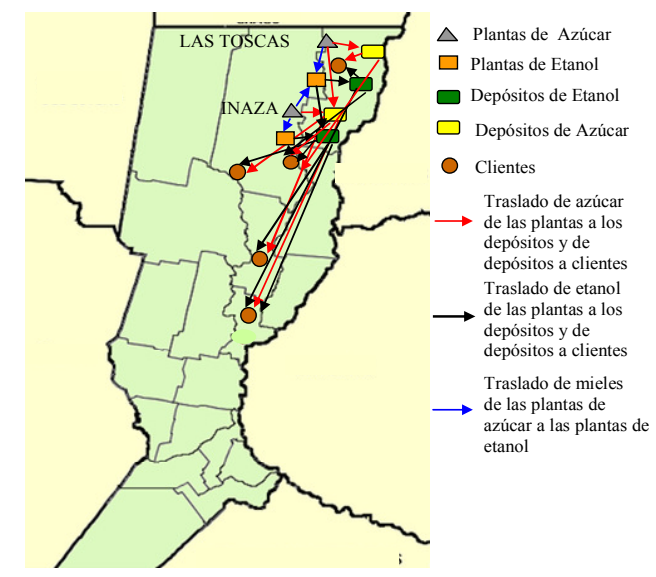

Fig. 3. Diseño óptimo de la CS para el caso de estudio

En esta primera solución, la CS incluye 2 plantas y depósitos de etanol, ubicados en las regiones 1 y 2 respectivamente, y 2 depósitos de azúcar, que se localizan junto con cada planta de azúcar instalada en el departamento General Obligado. Además, se abastece a todos los clientes.

La Tabla 5 muestra las cantidades de etanol y vinazas producidas en cada planta en cada período. Las vinazas producidas en ambas plantas son desechadas al río Paraná en cada uno de los períodos. El modelo determina además el número y tipo de 
vehículo utilizado entre los diferentes nodos de la CS. Por cuestiones de espacio, sólo se muestran los flujos y el número de camiones utilizados entre las plantas de etanol y sus depósitos (Tabla 6).

Tabla 5. Cantidades de etanol y vinazas producidas en cada planta (ton)

\begin{tabular}{lllll}
\hline Períodos & \multicolumn{2}{c}{ planta de la región 1 } & \multicolumn{2}{c}{ planta de la región 2 } \\
& etanol & vinazas & etanol & vinazas \\
\hline 1 & 389.216 & 5499.628 & 389.216 & 5499.628 \\
2 & 955.414 & 13500 & 600.918 & 8490.967 \\
3 & 194.608 & 2749.814 & 2749.814 & 2749.814 \\
4 & 194.608 & 2749.814 & 2749.814 & 2749.814 \\
\hline
\end{tabular}

Tabla 6. Etanol transportado desde cada planta a cada depósito (ton) y número de camiones utilizados de cada capacidad en cada período

\begin{tabular}{|c|c|c|c|c|}
\hline $\begin{array}{l}\text { Planta a } \\
\text { depósito }\end{array}$ & período 1 & período 2 & período 3 & período 4 \\
\hline $1-1$ & $\begin{array}{l}175 \text { ton } \\
9 \text { de } 20 \text { ton }\end{array}$ & $\begin{array}{l}780 \text { ton } \\
12 \text { de } 5 \text { ton } \\
30 \text { de } 10 \text { ton } \\
22 \text { de } 20 \text { ton }\end{array}$ & $\begin{array}{l}160.784 \text { ton } \\
9 \text { de } 20 \text { ton }\end{array}$ & $\begin{array}{l}0 \\
0\end{array}$ \\
\hline $1-2$ & $\begin{array}{l}214.216 \text { ton } \\
11 \text { de } 20 \text { ton }\end{array}$ & $\begin{array}{l}175.41 \text { ton } \\
9 \text { de } 20\end{array}$ & $\begin{array}{l}33.825 \text { ton } \\
2 \text { de } 20 \text { ton }\end{array}$ & $\begin{array}{l}194.608 \text { ton } \\
10 \text { de } 20 \text { ton }\end{array}$ \\
\hline $2-2$ & $\begin{array}{l}389.216 \text { ton } \\
20 \text { de } 20 \text { ton }\end{array}$ & $\begin{array}{l}600.918 \\
1 \text { de } 10 \text { ton } \\
30 \text { de } 20 \text { ton }\end{array}$ & $\begin{array}{l}194.608 \text { ton } \\
10 \text { de } 20 \text { ton }\end{array}$ & $\begin{array}{l}194.608 \text { ton } \\
10 \text { de } 20 \text { ton }\end{array}$ \\
\hline
\end{tabular}

Por último, se debe mencionar que el valor del daño total es igual a la cota superior del parámetro $\varepsilon$ debido a que las ganancias son maximizadas, lo cual trae aparejado producir más y por ende el daño causado al ambiente es mayor. Los valores para cada una de las etapas del LCA, correspondientes a la producción de azúcar y etanol y al transporte de azúcar, melazas, etanol y vinazas son presentados en la Tabla 7.

En la Tabla 7 se muestra que el impacto generado por las producciones de azúcar y etanol en los distintos períodos, alcanza un valor considerablemente mayor a los valores obtenidos por el impacto ocasionado por el transporte de materiales. Esto se debe a que ambos procesos de producción tienen varios residuos que son vertidos al medio ambiente, mientras que el daño provocado por el transporte es menor debido a las cortas distancias y a que los factores adoptados para el impacto ambiental son menores en esta categoría. Asimismo, los valores obtenidos para el impacto en la producción de etanol son más altos que los alcanzados en la producción de azúcar debido a que se producen grandes cantidades de vinazas (14.13 ton por 1 ton de etanol), las cuales son desechadas a los ríos.

Con el objetivo de encontrar diseños con menor impacto ambiental, se establecen cotas más ajustadas para $\varepsilon$. La Figura 4 muestra el conjunto de soluciones Pareto óptima. En todos los casos, la solución óptima consiste en producir la mayor cantidad de etanol y el daño ambiental toma el valor de la cota superior $\varepsilon_{\max }$. Por ejemplo, 
cuando el $\varepsilon=6000000$ instala una única planta de etanol en la región 3, pero ésta produce etanol sólo en el período 1. Cuando $\varepsilon=8000000$ instala sólo la planta de etanol de la región 3 pero produce en $\operatorname{los}$ períodos 1 y 3 , al igual que cuando $\varepsilon=10000000$, pero en este caso produce mayor cantidad en el período 3 . A medida que el valor de $\varepsilon_{\max }$ se incrementa, se produce más cantidad de etanol y obviamente el $V P N$ aumenta.

Tabla 7. Impacto de acuerdo a los principios del LCA $\left(\mathrm{kg} \mathrm{CO}_{2}\right)$

\begin{tabular}{lllllll}
\hline Períodos & $\begin{array}{l}\text { Impacto } \\
\text { generado } \\
\text { por la } \\
\text { prod. de } \\
\text { azúcar }\end{array}$ & $\begin{array}{l}\text { Impacto } \\
\text { generado } \\
\text { por la } \\
\text { prod. de } \\
\text { etanol }\end{array}$ & $\begin{array}{l}\text { Impacto } \\
\text { generado } \\
\text { por el } \\
\text { transp. de } \\
\text { azúcar }\end{array}$ & $\begin{array}{l}\text { Impacto } \\
\text { generado } \\
\text { por el } \\
\text { transp. de } \\
\text { melaza }\end{array}$ & $\begin{array}{l}\text { Impacto } \\
\text { generado } \\
\text { por el } \\
\text { transp. de } \\
\text { etanol }\end{array}$ & $\begin{array}{l}\text { Impacto } \\
\text { generado } \\
\text { por el } \\
\text { transp. de } \\
\text { vinaza }\end{array}$ \\
\hline 1 & 299960 & 7890400 & 812.7 & 1.2 & 278.8 & 379.2 \\
2 & 599920 & 15775000 & 817.4 & 31.0 & 154.2 & 725.2 \\
3 & 149980 & 3495200 & 1085.8 & 0.6 & 335.0 & 181.4 \\
4 & 149980 & 3495200 & 558.0 & 0.6 & 355.0 & 181.4 \\
\hline
\end{tabular}

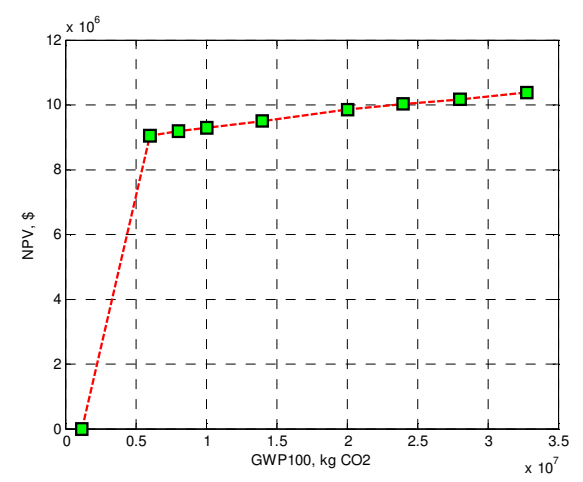

Fig. 4. Conjuntos de soluciones Pareto del GWP100 versus NPV.

\section{Conclusiones}

En este trabajo se desarrolló un MILP para el diseño óptimo de una CS para la producción de etanol a partir de melazas provenientes de la producción de azúcar de caña, con el fin de maximizar el valor presente neto y minimizar el daño ambiental generado por la producción de azúcar y etanol y por el transporte de azúcar, etanol, melaza y vinazas.

El enfoque de modelado propuesto y la estrategia de solución fueron aplicados a la producción de azúcar de la región centro-norte de la provincia de Santa Fe, con el 
objetivo de analizar la producción sustentable de bioetanol. Se analizaron las diferentes soluciones de Pareto y se evaluaron los diferentes escenarios con respecto al daño generado al medio ambiente en cada una de las etapas del LCA. Los resultados indican que a mayor ganancia, mayor es el impacto ambiental provocado, debido a la gran cantidad de residuos (vinazas) generada por la producción de etanol.

El enfoque propuesto representa una herramienta útil para adoptar diseños sustentables, como así también para evaluar la performance de ingenios y plantas de bioetanol ya instalados, y realizar las modificaciones necesarias para lograr esquemas sustentables.

Agradecimientos. Los autores agradecen a la Universidad Nacional del Litoral por el apoyo financiero recibido.

\section{Referencias}

1. Bioetanol de caña de azúcar: energía para el desarrollo sostenible / coordinación BNDES y CGEE. ISBN: 978-85-87545-26-8. Río de Janeiro: BNDES (2008).

2. Grossmann, I.E., Guillén-Gosálbez, G.: Scope for the application of mathematical programming techniques in the synthesis and planning of sustainable processes. Comp. and Chem. Engn., 34(9), 1365-1373 (2010).

3. Guillén-Gosálbez, G., Grossmann, I.E.: Optimal design and planning of sustainable chemical supply chains under uncertainty. AIChE Jounal, 55(1), 99-121 (2009).

4. Zamboni, A., Shah, N., Bezzo F.: Spatially Explicit Static Model for the Strategic Design of Future Bioethanol Production Systems. 1. Cost Minimization. Energy Fuels, 23 (10), 5121-5133 (2009).

5. Zamboni, A., Bezzo F., Shah, N.: Spatially Explicit Static Model for the Strategic Design of Future Bioethanol Production Systems. 2. Multi-Objective Environmental Optimization. Energy Fuels, 23 (10), 5134--5143 (2009).

6. Mele, F.D., Kostin A.M., Guillén-Gosálbez, G., Jiménez, L.: Multiobjective model for more suistainable suel supply chains. A case study of the sugar cane industry in Argentina. Ind. \& Eng. Chem. Res. 50 (9), 4939--4958 (2011).

7. Corsano, G., Vecchietti, A.R., Montagna J.M.: Optimal design for sustainable bioethanol supply chain considering detailed plant performance model. Comp. \& Chem. Engn., in press doi: 10.1016/j.compchemeng.2011.01.008, (2011).

8. Wang, F., Lai, X., Shi, N.: A multi-objective optimization for green supply chain network design. Decision Support Systems 51, 262--269 (2011).

9. Pinto-Varela, T., Barbosa-Póvoa,A., Novaisa A.: Bi-objective optimization approach to the design and planning of supply chains: Economic versus environmental performances. Comp. \& Chem. Engn., doi:10.1016/j.compchemeng.2011.03.009 (2011).

10. INTA Argentina, http://www.inta.gov.ar/info/bioenergia/bio.htm.

11. Brooke A, Kendrick D, Meeraus A, Raman R.: GAMS, A User Guide. Calif: Scientific press (1998). 